\title{
Prevalência e caracterização da anemia em idosos atendidos pela Estratégia Saúde da Família
}

\author{
Prevalence and characterization of anemia in elderly served by the Family Health Strategy
}

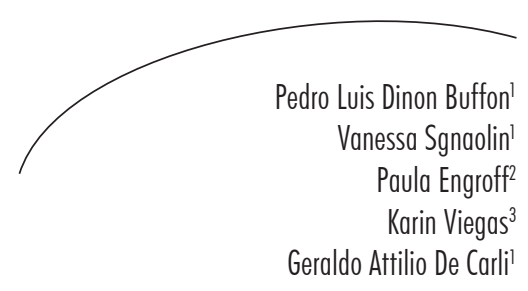

Resumo

Objetivo: Avaliar a prevalência de anemia em idosos atendidos pela Estratégia Saúde da Família do município de Porto Alegre-RS, Brasil, relacionando com as condições socioeconômicas e de saúde. Métodos: Estudo transversal exploratório observacional, no qual se realizaram coletas de informações epidemiológicas e de sangue. Os parâmetros hematológicos avaliados foram concentração de hemoglobina, volume corpuscular médio, concentração de hemoglobina corpuscular média e amplitude de distribuição das hemácias. Resultados: Foram avaliados 556 idosos. A prevalência de anemia, avaliada pela dosagem de hemoglobina, foi de $8,8 \%$, representando $10,1 \%$ para os homens e $8,1 \%$ para as mulheres. A anemia normocítica e normocrômica foi o tipo de anemia mais comumente encontrada (34,0\%), sugestiva de doença crônica. Além disso, a prevalência de anemia normocítica e hipocrômica foi de 32,0\%, sugerindo anemia por doença crônica nas fases iniciais de deficiência de ferro. $\mathrm{Na}$ análise das condições socioeconômicas e de saúde, diferenças significativas foram encontradas entre faixa etária, cor, escolaridade, presença ou não de cuidador principal, internação hospitalar, doenças da tireoide, relato de fraqueza e utilização de antianêmicos. Conclusão: Foi observada baixa prevalência de anemia nos idosos de Porto Alegre-RS. Inúmeros são os fatores desencadeantes dos baixos níveis de hemoglobina. Faixa etária, cor, renda mensal familiar, escolaridade, cuidados e doenças associadas são relevantes para influenciar nesse acontecimento. A anemia não deve ser considerada uma condição associada ao processo de envelhecimento, mas sim às inúmeras doenças, que são frequentes nessa população e, portanto, merece atenção clínica adequada.

\footnotetext{
Pontifícia Universidade Católica do Rio Grande do Sul, Instituto de Geriatria e Gerontologia, Programa de Pós-graduação em Gerontologia Biomédica. Porto Alegre, RS, Brasil.

2 Pontifícia Universidade Católica do Rio Grande do Sul, Hospital São Lucas, Instituto de Geriatria e Gerontologia, Laboratório de Bioquímica, Genética Molecular e Parasitologia. Porto Alegre, RS, Brasil.

3 Universidade Federal de Ciências da Saúde de Porto Alegre, Faculdade de Enfermagem. Porto Alegre, RS, Brasil.
}

Financiamento: Fundação de Amparo à Pesquisa do Estado do Rio Grande do Sul - processo $\mathrm{n}^{\circ}$ 09/0055-0/2009.

Palavras-chave: Anemia. Idoso. Inquéritos Epidemiológicos. Índices de Eritrócitos. Fatores Socioeconômicos. 


\section{Abstract}

Objective: To evaluate the prevalence of anemia among elderly people related to socioeconomic and health conditions in elderly attended by the Family Health Strategy (FHS) in the city of Porto Alegre-RS, Brazil. Methods: Observational, cross-sectional and exploratory study in which epidemiological information, and blood sampling. The hematological parameters evaluated were hemoglobin concentration, mean corpuscular volume, mean corpuscular hemoglobin concentration and red cell distribution width. Results: We assessed 556 senior citizens. The prevalence of anemia, assessed by hemoglobin measurement was $8.8 \%$, representing $10.1 \%$ for men and $8.1 \%$ for women. A normochromic normocytic anemia was the type of anemia most commonly found (34.0\%), suggesting chronic disease. Furthermore, the prevalence of normocytic and hypochromic anemia was $32.0 \%$, suggesting an evolution of anemia by chronic disease in early stages of iron deficiency. Analyzing health and socioeconomic conditions, significant differences were found among age range, color, educational level, presence or absence of primary caregiver, hospitalization, thyroid disease, report of weakness and use of anti-anemic medications. Conclusion: Low prevalence of anemia in the elderly in Porto Alegre-RS was observed. Numerous are the triggering factors of low hemoglobin levels. Factors such as age, color, family income, education, care, associated diseases are relevant to influence this event. Anemia should not be considered only associated with aging condition, but to many diseases that are common in this population and therefore deserves appropriate medical attention. Characterization of anemia should be carefully performed to aid its correct treatment.
Key words: Anemia. Elderly. Health Surveys. Erythrocyte Indices. Socioeconomic Factors.

\section{INTRODUÇÃ̃o}

O envelhecimento vem se tornando um assunto bastante debatido devido à maior longevidade dos indivíduos. A proporção da pirâmide populacional vem se modificando à medida que o tempo passa por causa do aumento da população idosa em relação à população total, bem como por avanços na medicina e melhoria da qualidade de vida. Em paralelo a essas modificações, doenças próprias do envelhecimento ganham maior expressão no conjunto da sociedade. A diminuição progressiva da capacidade fisiológica, associada a outras doenças, faz com que os profissionais da área da saúde tenham que se aprimorar no que diz respeito à saúde do idoso.

Segundo a Organização Mundial da Saúde (OMS), a anemia é definida como a diminuição da concentração de hemoglobina circulante no sangue, sendo desencadeada por mecanismos fisiopatológicos diversos. A redução da concentração de hemoglobina é considerada patológica quando apresenta valores abaixo de $12,0 \mathrm{~g} / \mathrm{dL}$ para mulheres e de 13,0 $\mathrm{g} / \mathrm{dL}$ para homens. Estimativas da OMS projetam que mais de dois bilhões de pessoas no mundo são anêmicas, porém nos idosos a anemia é o problema hematológico mais comumente encontrado, estando associada com o aumento do risco de mortalidade e morbidade, assim como na redução da qualidade de vida. ${ }^{1}$

Estudo realizado por Beghé et al. ${ }^{2}$ relatou variação na prevalência de anemia de $2,9 \%$ a $61,0 \%$ em homens e de $3,3 \%$ a $41,0 \%$ em mulheres, podendo ser justificada pelo tipo de estudo, estado de saúde dos idosos e os critérios para a definição conceitual de anemia. Quanto à etiologia, pode-se considerar que em um mesmo idoso, pode se fazer presente mais de um fator desencadeante da anemia, tais como carência nutricional, uso de medicamentos, doenças crônicas, entre outras..$^{3-5}$ Diante desse cenário, há necessidade de estudos com o objetivo de avaliar a prevalência de anemia em idosos, relacionando com condições gerais dos mesmos, 
a fim de avaliar sua relação com distúrbios hematopoiéticos frequentes nessa faixa etária.

Este estudo teve como objetivo avaliar a prevalência de anemia em idosos atendidos pela Estratégia Saúde da Família do município de Porto Alegre-RS, Brasil, relacionada às condições socioeconômicas e de saúde.

\section{MÉTODOS}

Delineamento

Estudo do tipo transversal, exploratório e observacional de base populacional para os eventos mais frequentes na população idosa do município de Porto Alegre-RS. A pesquisa faz parte do "Estudo Epidemiológico e Clínico dos Idosos atendidos pela Estratégia Saúde da Família do município de Porto Alegre", realizado pelo Instituto de Geriatria e Gerontologia da Pontifícia Universidade Católica do Rio Grande do Sul (PUCRS), em parceria com a Secretaria Municipal de Saúde de Porto Alegre, no período de março de 2011 e dezembro de 2012.

\section{População em estudo}

A população estudada compreendeu indivíduos de ambos os sexos, com idade $\geq 60$ anos, residentes no município de Porto Alegre-RS e cadastrados nas regiões adstritas da Estratégia Saúde da Família (ESF).

Considerando uma população-alvo de 22 mil idosos atendidos pela ESF em Porto Alegre, foi definido um tamanho amostral mínimo de 540 indivíduos, considerando $2,5 \%$ de erro aceitável para uma prevalência de anemia esperada de $10,0 \%$.

Para a seleção inicial desses idosos, e já prevendo possíveis perdas durante o desenvolvimento do estudo, foram sorteadas 30 equipes da ESF de forma estratificada por gerência distrital (GD). De cada equipe, foram sorteados 36 idosos, totalizando 1.080 idosos.
Coleta de dados

Para as entrevistas, os idosos receberam a visita, em suas residências, de agentes comunitários de saúde (ACS), que explicaram os objetivos da pesquisa e convidaram o idoso a participar. Após, o idoso ou cuidador respondeu a um questionário geral contendo dados socioeconômicos e condições de saúde.

As coletas das amostras sanguíneas foram realizadas na sede da ESF ou em suas residências (no caso de idosos acamados) pela equipe do projeto, por meio da obtenção de uma alíquota de aproximadamente $4 \mathrm{ml}$ de sangue total em tubo contendo o anticoagulante ácido etilenodiamino tetra-acético (EDTA). As amostras foram encaminhadas ao Laboratório de Patologia Clínica do Hospital São Lucas, da PUCRS, para realização do hemograma.

Parâmetros laboratoriais

O hemograma foi realizado no Setor de Hematologia do Laboratório de Patologia Clínica do Hospital São Lucas da PUCRS, nos equipamentos de automação Sysmex 2100 e Sysmex XE 5000 (Sysmex, Kobe, Japan). Os parâmetros avaliados foram hemoglobina e índices hematimétricos - volume corpuscular médio (VCM), concentração de hemoglobina corpuscular média (CHCM) e amplitude de distribuição das hemácias (RDW).

Os valores de referência da hemoglobina para homens é 13,0g/dL e para mulheres 12,0g/dL, VCM 80 - 100 fL, CHCM 31,0 - 35,0 g/dL e RDW até $15,0 \%{ }^{1}$

Análise estatística

O banco de dados foi estruturado em Excel e, posteriormente, analisado por meio do programa Statistical Package for Social Science (SPSS) versão 17.0. Para a análise bivariada entre anemia e as variáveis categóricas, foi utilizado o teste 
Qui-quadrado de Pearson, sendo que nas tabelas de contingência em que, pelo menos, $25 \%$ dos valores das células apresentaram frequência esperada menor do que 5 foi utilizado o teste Exato de Fisher. Para as variáveis contínuas, quando a comparação ocorreu entre dois grupos independentes, foi aplicado o teste $t$ Student.

\section{Considerações éticas}

O estudo foi aprovado pelos Comitês de Ética em Pesquisa (CEP) da Pontifícia Universidade Católica do Rio Grande do Sul (parecer $\mathrm{n}^{\mathrm{o}}$ 10/04967-2010) e da Secretaria Municipal de Saúde de Porto Alegre (processo $\mathrm{n}^{\mathrm{o}}$ 001.021434.10.7/2010), atendendo às diretrizes e normas regulamentadoras em pesquisa. $\mathrm{O}$ Termo de Consentimento Livre e Esclarecido foi obtido de todos os participantes.

\section{RESULTADOS}

A amostra compreendeu 556 indivíduos, sendo $64,4 \%(\mathrm{n}=358)$ mulheres e $35,6 \%(\mathrm{n}=198)$

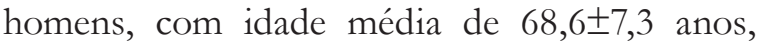
mínima de 60 anos e máxima de 103 anos.

A prevalência de anemia foi de $8,8 \%(\mathrm{n}=49)$, com intervalo de confiança de $95 \%$ variando de 6,3 a 11,7\%. Embora a população fosse predominantemente feminina, os homens apresentaram prevalência de anemia maior $(10,1 \%)$ do que as mulheres $(8,1 \%)$.

Quando comparadas as idades entre os grupos com e sem anemia, a média foi significantemente mais elevada entre os investigados com anemia $72,7 \pm 9,3$ anos, do que nos sem anemia $68,2 \pm 7,0$ anos $(p<0,05)$.

Em função da diferença significativa entre as médias de idade, foram criadas faixas etárias, buscando identificar de forma detalhada as diferenças mais expressivas. Os resultados apontaram que na faixa etária de 80 anos ou mais os idosos apresentaram alta prevalência de anemia - 24,5\% ( $n=12)$-, enquanto que no grupo sem anemia a maioria dos idosos pertencia à faixa etária de 60 a 69 anos - 93,7\% (n=328; $p<0,001)$. Estimou-se, ainda, o risco relativo para desenvolver anemia no grupo de 80 anos ou mais, que foi 3,1 vezes maior (IC95\%: 1,3-7,1) que as demais faixas etárias. Na comparação da cor, a associação estatisticamente significativa se configurou apontando que os idosos da cor preta apresentavam mais anemia, $16,2 \%(\mathrm{n}=17$; $p=0,007)$, segundo a tabela 1 .

$\mathrm{Na}$ avaliação da presença de cuidador principal, foi observada diferença estatística significativa, mostrando que os idosos que possuíam cuidador principal apresentaram maior prevalência de anemia - 14,4\% $(n=29$; $p=0,001)$. Em relação à renda mensal familiar, houve uma tendência sugerindo que os idosos que pertenciam às famílias com renda de até um salário mínimo apresentavam maior prevalência de anemia - 12,2\% ( $\mathrm{n}=20 ; p=0,054)$. Quanto à variável "escolaridade", verificou-se que os idosos com baixa escolaridade tiveram resultado significativo $(p=0,041)$, demonstrando que $9,7 \% \quad(\mathrm{n}=45) \quad$ desses apresentaram anemia (tabela 1). 
Tabela 1. Prevalência de anemia em idosos de acordo com os dados socioeconômicos. Porto Alegre, RS, 2012.

\begin{tabular}{|c|c|c|c|c|c|c|c|}
\hline \multirow{3}{*}{ Variáveis } & & & \multicolumn{4}{|c|}{ Anemia** } & \multirow{3}{*}{$p$} \\
\hline & \multicolumn{2}{|c|}{ Total $(\mathrm{n}=556)^{*}$} & \multicolumn{2}{|c|}{$\operatorname{Sim}(M<13$ e $F<12)$} & \multicolumn{2}{|c|}{ Não $(M \geq 13$ e $F \geq 12)$} & \\
\hline & $\mathrm{n}$ & $\%$ & $\mathrm{n}$ & $\%$ & $\mathrm{n}$ & $\%$ & \\
\hline \multicolumn{8}{|l|}{ Sexo* } \\
\hline Feminino & 358 & 64,4 & 29 & 8,1 & 329 & 91,9 & \multirow{2}{*}{$0,552 \Phi$} \\
\hline Masculino & 198 & 35,6 & 20 & 10,1 & 178 & 89,9 & \\
\hline \multicolumn{8}{|l|}{ Faixa etária } \\
\hline De 60 a 69 & 350 & 62,9 & 22 & 6,3 & 328 & 93,7 & \multirow{3}{*}{$<0,0019$} \\
\hline De 70 a 79 & 157 & 28,2 & 15 & 9,6 & 142 & 90,4 & \\
\hline 80 anos ou mais & 49 & 8,8 & 12 & 24,5 & 37 & 75,5 & \\
\hline \multicolumn{8}{|l|}{ Cor } \\
\hline Branca & 364 & 65,9 & 22 & 6,0 & 342 & 94,0 & \multirow{4}{*}{$0,007 \Phi$} \\
\hline Preta & 105 & 19,0 & 17 & 16,2 & 88 & 83,8 & \\
\hline Parda & 69 & 12,5 & 9 & 13,0 & 60 & 87,0 & \\
\hline Outros & 14 & 2,5 & 1 & 7,1 & 13 & 92,9 & \\
\hline \multicolumn{8}{|l|}{ Estado civil } \\
\hline Casado & 216 & 39,1 & 21 & 9,7 & 195 & 90,3 & \multirow{4}{*}{$0,648 \Phi$} \\
\hline Solteiro & 92 & 16,7 & 6 & 6,5 & 86 & 93,5 & \\
\hline Viúvo & 156 & 28,3 & 16 & 10,3 & 140 & 89,7 & \\
\hline Separado/desquitado & 88 & 15,9 & 6 & 6,8 & 82 & 93,2 & \\
\hline \multicolumn{8}{|l|}{ Tem cuidador principal } \\
\hline Não & 335 & 62,5 & 19 & 5,7 & 316 & 94,3 & \multirow{2}{*}{$0,001 \Phi$} \\
\hline $\operatorname{Sim}$ & 201 & 37,5 & 29 & 14,4 & 172 & 85,6 & \\
\hline \multicolumn{8}{|l|}{ Renda mensal (familiar) } \\
\hline Até um salário mínimo & 164 & 34,2 & 20 & 12,2 & 144 & 87,8 & \multirow{3}{*}{$0,054 €$} \\
\hline Até três salários mínimos & 265 & 55,2 & 22 & 8,3 & 243 & 91,7 & \\
\hline $\begin{array}{l}\text { Seis ou mais salários } \\
\text { mínimos }\end{array}$ & 51 & 10,6 & 2 & 3,9 & 49 & 96,1 & \\
\hline \multicolumn{8}{|l|}{ Escolaridade } \\
\hline Até $1^{\circ}$ grau incompleto & 464 & 84,5 & 45 & 9,7 & 419 & 90,3 & \multirow{2}{*}{$0,041 \Phi$} \\
\hline $1^{\circ}$ grau completo ou mais & 85 & 15,5 & 3 & 3,5 & 82 & 96,5 & \\
\hline
\end{tabular}

M: masculino. F: feminino. Salário mínimo no período do estudo R \$540,00.

*Percentuais obtidos com base no total da amostra; **Percentuais obtidos com base no total de cada categoria da anemia; $\Phi=$ teste Quiquadrado de Pearson; $\mathbb{q}=$ teste Exato de Fisher por simulação de Monte Carlo; $\boldsymbol{€}=$ teste de Tendência Linear do Qui-quadrado. 
$\mathrm{Na}$ análise das variáveis relacionadas aos dados de saúde (tabela 2), os idosos que consultaram médico nos últimos seis meses apresentavam uma tendência, indicando que $9,4 \%(n=45)$ dos idosos com anemia procuraram por serviços médicos $(p=0,064)$. E 18,2\% $(\mathrm{n}=18)$ dos idosos que tiveram algum tipo de internação hospitalar no último ano apresentaram anemia $(p<0,001)$. Dos idosos com anemia, houve associação significativa quando existiu relato de doença da tireoide - 20,4\% ( $\mathrm{n}=10)$, e fraqueza - $11,9 \% \quad(\mathrm{n}=31 ; p<0,05)$. As demais doenças não apresentaram resultados estatisticamente significativos quando comparadas com a presença de anemia, porém quando analisadas as doenças hepáticas separadamente, observouse resultado significativo para os idosos com cirrose hepática $-33,3 \%(\mathrm{n}=2 ; p=0,039)$.

Tabela 2. Prevalência de anemia em idosos de acordo com os dados de saúde. Porto Alegre-RS, 2012.

\begin{tabular}{|c|c|c|c|c|c|c|c|}
\hline \multirow{3}{*}{ Variáveis } & & & \multicolumn{4}{|c|}{ Anemia** } & \multirow{3}{*}{$p$} \\
\hline & \multicolumn{2}{|c|}{ Total $(\mathrm{n}=556)^{*}$} & \multicolumn{2}{|c|}{$\operatorname{Sim}(M<13$ e $F<12)$} & \multicolumn{2}{|c|}{ Não $(M \geq 13$ e $F \geq 12)$} & \\
\hline & $\mathrm{n}$ & $\%$ & $n$ & $\%$ & $\mathrm{n}$ & $\%$ & \\
\hline \multicolumn{8}{|c|}{ Autopercepção de saúde } \\
\hline Ótima/boa & 195 & 35,3 & 16 & 8,2 & 179 & 91,8 & \multirow{3}{*}{$0,583 €$} \\
\hline Regular & 303 & 54,9 & 26 & 8,6 & 277 & 91,7 & \\
\hline Má/péssima & 54 & 9,8 & 6 & 11,1 & 48 & 88,9 & \\
\hline \multicolumn{8}{|c|}{$\begin{array}{l}\text { Consultou médico nos } \\
\text { últimos seis meses }\end{array}$} \\
\hline Não & 71 & 12,9 & 2 & 2,8 & 69 & 97,2 & \multirow{2}{*}{$0,064 \Phi$} \\
\hline $\operatorname{Sim}$ & 479 & 87,1 & 45 & 9,4 & 434 & 90,6 & \\
\hline \multicolumn{8}{|c|}{ Internou no último ano } \\
\hline Não & 453 & 82,1 & 29 & 6,4 & 424 & 93,6 & \multirow{2}{*}{$<0,001 \Phi$} \\
\hline $\operatorname{Sim}$ & 99 & 17,9 & 18 & 18,2 & 81 & 81,8 & \\
\hline \multicolumn{8}{|c|}{ Doenças cardiovasculares } \\
\hline Não & 121 & 21,8 & 11 & 9,1 & 110 & 90,9 & \multirow{2}{*}{$0,903 \Phi$} \\
\hline $\operatorname{Sim}$ & 435 & 78,2 & 38 & 8,7 & 397 & 91,3 & \\
\hline \multicolumn{8}{|c|}{ Doenças cerebrovasculares } \\
\hline Não & 455 & 85,4 & 38 & 8,4 & 417 & 91,6 & \multirow{2}{*}{$0,359 \Phi$} \\
\hline $\operatorname{Sim}$ & 78 & 14,6 & 9 & 11,5 & 69 & 88,5 & \\
\hline \multicolumn{8}{|l|}{ Doenças hepáticas } \\
\hline Não & 477 & 90 & 40 & 8,4 & 437 & 91,6 & \multirow{2}{*}{$0,106 \Phi$} \\
\hline $\operatorname{Sim}$ & 53 & 10 & 8 & 15,1 & 45 & 84,9 & \\
\hline
\end{tabular}




\begin{tabular}{|c|c|c|c|c|c|c|c|}
\hline \multirow{3}{*}{ Variáveis } & & & \multicolumn{4}{|c|}{ Anemia** } & \multirow{3}{*}{$p$} \\
\hline & \multicolumn{2}{|c|}{ Total $(\mathrm{n}=556)^{*}$} & \multicolumn{2}{|c|}{$\operatorname{Sim}(M<13$ e $F<12)$} & \multicolumn{2}{|c|}{ Não $(M \geq 13$ e $F \geq 12)$} & \\
\hline & $\mathrm{n}$ & $\%$ & $\mathrm{n}$ & $\%$ & $\mathrm{n}$ & $\%$ & \\
\hline \multicolumn{8}{|l|}{ Doenças ósseas } \\
\hline Não & 324 & 61,2 & 28 & 8,6 & 296 & 91,4 & \multirow{2}{*}{$0,664 \Phi$} \\
\hline $\operatorname{Sim}$ & 205 & 38,8 & 20 & 9,8 & 185 & 90,2 & \\
\hline \multicolumn{8}{|l|}{ Doenças renais } \\
\hline Não & 468 & 89,8 & 40 & 8,5 & 428 & 91,5 & \multirow{2}{*}{$0,262 \Phi$} \\
\hline $\operatorname{Sim}$ & 53 & 10,2 & 7 & 13,2 & 46 & 86,8 & \\
\hline \multicolumn{8}{|l|}{ Doença da tireoide } \\
\hline Não & 482 & 90,8 & 36 & 7,5 & 446 & 92,5 & \multirow{2}{*}{$0,002 \Phi$} \\
\hline $\operatorname{Sim}$ & 49 & 9,2 & 10 & 20,4 & 39 & 79,6 & \\
\hline \multicolumn{8}{|l|}{ Diabetes } \\
\hline Não & 379 & 71,2 & 29 & 7,7 & 350 & 92,3 & \multirow{2}{*}{$0,199 \Phi$} \\
\hline $\operatorname{Sim}$ & 153 & 28,8 & 17 & 11,1 & 136 & 88,9 & \\
\hline \multicolumn{8}{|l|}{ Câncer } \\
\hline Não & 465 & 88,1 & 38 & 8,2 & 427 & 91,8 & \multirow{2}{*}{$0,110 \Phi$} \\
\hline Sim & 63 & 11,9 & 9 & 14,3 & 54 & 85,7 & \\
\hline \multicolumn{8}{|l|}{$\begin{array}{l}\text { Doenças } \\
\text { neuropsiquiátricas }\end{array}$} \\
\hline Não & 335 & 62,2 & 29 & 8,7 & 306 & 91,3 & \multirow{2}{*}{$0,795 \Phi$} \\
\hline $\operatorname{Sim}$ & 204 & 37,8 & 19 & 9,3 & 185 & 90,7 & \\
\hline \multicolumn{8}{|c|}{ Fraqueza (falta de força) } \\
\hline Não & 281 & 51,8 & 16 & 5,7 & 265 & 94,3 & \multirow{2}{*}{$0,011 \Phi$} \\
\hline $\operatorname{Sim}$ & 261 & 48,2 & 31 & 11,9 & 230 & 88,1 & \\
\hline
\end{tabular}

$\mathrm{M}=$ masculino; $\mathrm{F}=$ feminino; * percentuais obtidos com base no total da amostra; ** percentuais obtidos com base no total de cada categoria da anemia; $\Phi=$ teste Qui-quadrado de Pearson; $€=$ teste de Tendência Linear do Qui-quadrado.

Ainda na avaliação do tabagismo, alcoolismo (tabela 3) e condições higiênicas e sanitárias, como possuir caixa d' água, banheiro e filtro de água em suas residências, não foram verificados resultados estatisticamente significativos quando relacionados com a prevalência de anemia.
Dos idosos que utilizam medicamentos antianêmicos, $\quad 37,5 \% \quad(n=3) \quad$ apresentaram anemia $(p=0,004)$. Os demais medicamentos que poderiam estar associados à presença de anemia não apresentaram significância estatística (tabela 4). 
Tabela 3. Prevalência de anemia em idosos de acordo com o tabagismo e consumo de álcool. Porto Alegre-RS, 2012.

\begin{tabular}{|c|c|c|c|c|c|c|c|}
\hline \multirow{3}{*}{ Variáveis } & \multirow{2}{*}{\multicolumn{2}{|c|}{$\begin{array}{c}\text { Total } \\
(\mathrm{n}=556)^{*}\end{array}$}} & \multicolumn{4}{|c|}{ Anemia** } & \multirow{3}{*}{$p$} \\
\hline & & & \multicolumn{2}{|c|}{$\operatorname{Sim}(M<13$ e $F<12)$} & \multicolumn{2}{|c|}{ Não $(M \geq 13$ e $F \geq 12)$} & \\
\hline & $\mathrm{n}$ & $\%$ & $\mathrm{n}$ & $\%$ & $\mathrm{n}$ & $\%$ & \\
\hline \multicolumn{8}{|l|}{ Hábito de fumar cigarro } \\
\hline $\operatorname{Sim}$ & 111 & 20,6 & 4 & 3,6 & 107 & 96,4 & \multirow{3}{*}{$0,090 \Phi$} \\
\hline Não atualmente, mas já fumou & 203 & 37,6 & 21 & 10,3 & 182 & 89,7 & \\
\hline Não nunca fumou & 226 & 41,9 & 23 & 10,2 & 89,8 & 41,3 & \\
\hline \multicolumn{8}{|l|}{ Consome bebida alcoólica } \\
\hline Não & 376 & 71,6 & 33 & 8,8 & 343 & 91,2 & \multirow[t]{2}{*}{$0,790 \Phi$} \\
\hline Sim & 149 & 28,4 & 12 & 8,1 & 137 & 91,9 & \\
\hline
\end{tabular}

$\mathrm{M}=$ masculino; $\mathrm{F}=$ feminino; $*$ percentuais obtidos com base no total da amostra; **percentuais obtidos com base no total de cada categoria da anemia; $\Phi=$ teste Qui-quadrado de Pearson.

Tabela 4. Prevalência de anemia em idosos de acordo com o uso de medicamentos. Porto Alegre-RS, 2012.

\begin{tabular}{|c|c|c|c|c|c|c|c|}
\hline \multirow{3}{*}{ Variáveispp } & \multirow{2}{*}{\multicolumn{2}{|c|}{ Total $(\mathrm{n}=556)^{*}$}} & \multicolumn{4}{|c|}{ Anemia** } & \multirow{3}{*}{$p$} \\
\hline & & & \multicolumn{2}{|c|}{$\operatorname{Sim}(M<13$ e $F<12)$} & \multicolumn{2}{|c|}{ Não $(M \geq 13$ e $F \geq 12)$} & \\
\hline & $\mathrm{n}$ & $\%$ & $\mathrm{n}$ & $\%$ & $\mathrm{n}$ & $\%$ & \\
\hline \multicolumn{8}{|c|}{ Utiliza medicamentos } \\
\hline Não & 58 & 10,8 & 4 & 6,9 & 54 & 93,1 & \multirow{2}{*}{$0,570 \Phi$} \\
\hline Sim & 481 & 89,2 & 44 & 9,1 & 437 & 90,9 & \\
\hline \multicolumn{8}{|c|}{$\begin{array}{l}\text { Anti-inflamatório não } \\
\text { esteroidal (AINES) }\end{array}$} \\
\hline Não & 492 & 88,5 & 44 & 8,9 & 448 & 91,1 & \multirow{2}{*}{$0,764 \Phi$} \\
\hline Sim & 64 & 11,5 & 5 & 7,8 & 59 & 92,2 & \\
\hline \multicolumn{8}{|l|}{ Antianêmicos } \\
\hline Não & 548 & 98,6 & 46 & 8,4 & 502 & 91,6 & \multirow{2}{*}{$0,004 \Phi$} \\
\hline Sim & 8 & 1,4 & 3 & 37,5 & 5 & 62,5 & \\
\hline
\end{tabular}

$\mathrm{M}=$ masculino; $\mathrm{F}=$ feminino; $*$ percentuais obtidos com base no total da amostra; ** percentuais obtidos com base no total de cada categoria da anemia; $\Phi=$ teste Qui-quadrado de Pearson.

Pormeio da análise dosíndices hematimétricos, CHCM, VCM e RDW, foram realizadas avaliação morfológica eritrocitária e classificação das anemias (tabela 5). Nos idosos com anemia, as alterações mais encontradas foram: hipocromia isolada em 32,0\% ( $\mathrm{n}=16)$, hipocromia e anisocitose em 10,0\% ( $\mathrm{n}=5)$ e hipocromia, microcitose e anisocitose em 6,0\% ( $\mathrm{n}=3)$. Em 34,0\% ( $\mathrm{n}=17)$ dos idosos anêmicos, não foi relatada nenhuma alteração nos índices hematimétricos. 
Tabela 5. Distribuição da frequência das alterações eritrocitárias nos idosos com e sem anemia. Porto Alegre-RS, 2012.

\begin{tabular}{|c|c|c|c|c|c|c|c|c|}
\hline \multicolumn{3}{|c|}{ Alterações eritrocitárias } & \multicolumn{6}{|c|}{ Anemia } \\
\hline \multirow[b]{2}{*}{$\mathrm{CHCM}$} & \multirow[b]{2}{*}{ VCM } & \multirow[b]{2}{*}{ RDW } & \multicolumn{3}{|c|}{$\operatorname{Sim}(n=49)$} & \multicolumn{3}{|c|}{ Não (n=507) } \\
\hline & & & $\mathrm{n}$ & $\%$ & $\mathrm{IC}(95 \%)$ & $\mathrm{n}$ & $\%$ & $\mathrm{IC}(95 \%)$ \\
\hline \multirow{6}{*}{ Hipocrômico $(\mathrm{CHCM}<32)$} & Microcitose & $<15$ & 1 & 2 & $0,3-3,7$ & 1 & 0,2 & $0,0-0,9$ \\
\hline & $(\mathrm{VCM}<80)$ & $\geq 15$ & 3 & 6 & $3,3-8,7$ & 1 & 0,2 & $0,0-0,9$ \\
\hline & Normocitose & $<15$ & 16 & 32 & $27,7-36,3$ & 74 & 14,6 & $13,3-15,9$ \\
\hline & $(80-100)$ & $\geq 15$ & 5 & 10 & $7,8-12,2$ & 11 & 2,2 & $1,7-2,7$ \\
\hline & Macrocitose & $<15$ & 2 & 4 & $2,1-5,9$ & 1 & 0,2 & $0,0-0,9$ \\
\hline & $(\mathrm{VCM}>100)$ & $\geq 15$ & 0 & 0 & $0,0-4,6$ & 2 & 0,4 & $0,0-1,1$ \\
\hline \multirow{6}{*}{ Normocromia $(\mathrm{CHCM} \geq 32)$} & Microcitose & $<15$ & 1 & 2 & $0,3-3,7$ & 4 & 0,8 & $0,1-1,6$ \\
\hline & $(\mathrm{VCM}<80)$ & $\geq 15$ & 0 & 0 & $0,0-4,6$ & 2 & 0,4 & $0,0-1,1$ \\
\hline & Normocitose & $<15$ & 17 & 34 & $30,2-37,8$ & 383 & 75,5 & $73,0-78,0$ \\
\hline & $(80-100)$ & $\geq 15$ & 3 & 6 & $3,3-8,7$ & 13 & 2,6 & $2,3-2,9$ \\
\hline & Macrocitose & $<15$ & 1 & 2 & $0,3-3,7$ & 13 & 2,6 & $2,3-2,9$ \\
\hline & $(\mathrm{VCM}>100)$ & $\geq 15$ & 0 & 0 & $0,0-4,6$ & 2 & 0,4 & $0,0-0,9$ \\
\hline
\end{tabular}

$\mathrm{CHCM}=$ concentração de hemoglobina corpuscular média; $\mathrm{VCM}=$ volume corpuscular médio;

$\mathrm{RDW}=$ amplitude de distribuição das hemácias.

\section{DISCUSSÃO}

A anemia representa um problema clínico relevante na população idosa, com ampla repercussão na qualidade de vida e aumento da morbidade. ${ }^{6}$ Dados referentes à prevalência de anemia são discrepantes e podem estar relacionados às condições socioeconômicas.

O presente estudo encontrou prevalência de anemia de 8,8\% nos idosos residentes no município de Porto Alegre-RS, Brasil. A prevalência observada foi similar àquela demonstrada em estudo com idosos coreanos $(8,3 \%)$ e, ainda, menor do que a relatada na população idosa dos Estados Unidos (10,6\%). ${ }^{6,7}$ Em 2006, estudo realizado por Sgnaolin et al. ${ }^{8}$ também com idosos de Porto Alegre, encontrou prevalência de anemia de 12,8\%. A baixa prevalência identificada se deve provavelmente a uma boa cobertura de assistência à saúde dada a essa população, uma vez que a busca pela amostra foi realizada nas ESFs, que consistem em um programa que visa a promoção e prevenção da saúde. Dessa maneira, a assistência dada por toda a equipe de saúde, principalmente o ACS, é essencial para identificar possíveis alterações nas fases iniciais das doenças no idoso. ${ }^{9}$

Em relação à faixa etária, verifica-se que quanto maior a idade, maior a prevalência de anemia. Outros estudos realizados também demonstraram que a prevalência de anemia é menor nas faixas etárias abaixo dos 80 anos. ${ }^{8,10}$ A elevação da prevalência de anemia com o aumento da idade pode estar associada ao processo do envelhecimento, visto que há um decréscimo na produção de células sanguíneas. Assim como o envelhecimento, também está associado ao aumento de doenças crônicas e estas podem contribuir para o desenvolvimento da anemia. Por isso, é importante a adequada 
investigação das causas da anemia, a fim de realizar o diagnóstico precoce e o tratamento correto, promovendo melhores condições clínicas para os idosos. ${ }^{11}$

Quanto à variável cor, foi verificado que os idosos que se autorrelataram da cor preta apresentaram maior prevalência de anemia. Esse dado pode ser explicado pela associação verificado entre anemia e piores condições socioeconômicas, demográficas e de saúde, também descritas por Haverkate et al. ${ }^{12}$

Os idosos que tinham cuidador apresentaram prevalência de anemia maior do que aqueles que não tinham. Esse achado, possivelmente, está associado ao fato de que os idosos que necessitam da assistência de um cuidador apresentam-se debilitados por um maior comprometimento à saúde, devido à presença de inúmeras doenças crônicas. ${ }^{13}$

Níveis baixos de escolaridade foram associados à maior prevalência de anemia. $\mathrm{Na}$ amostra estudada, a baixa escolaridade (até $1^{\circ}$ grau incompleto) correspondia a 84,5\% dos indivíduos. Esse resultado é compatível com dados do Instituto Brasileiro de Geografia e Estatística (IBGE), que classificam a Região Sul em segundo lugar no índice de pessoas que não frequentaram a escola. ${ }^{14} \mathrm{~A}$ desigualdade associada à escolaridade indica diferente compreensão em relação a saúde e, consequentemente, na procura por serviços de saúde. Conforme dados do estudo realizado por Noronha \& Andrade, ${ }^{15}$ há uma desigualdade social em saúde a favor dos grupos com maior escolaridade.

Na avaliação dos dados de saúde, a maioria dos idosos que apresentou anemia esteve internada no último ano. Esses resultados sugerem que idosos internados estão em uma condição mais debilitada, devido às múltiplas doenças crônicas associadas, que podem levar ao desenvolvimento de anemia. ${ }^{11}$ Entre as patologias relatadas, a doença da tireoide apresentou maior índice de idosos anêmicos, achado que é confirmado pelas várias implicações que ocorrem na evolução da tireoideopatia (principalmente hipotireodismo), podendo ocasionar anemia. ${ }^{16}$ Entretanto, o mecanismo de interferência da tireoide na eritropoiese é controverso, existindo afirmativas que relacionam a anemia como uma simples adaptação fisiológica ao decréscimo da demanda de oxigênio, e outras a processos mais complexos, como a indiferenciação eritroide, a redução da ação do ácido retinoico no programa de diferenciação eritroide e a interferência na síntese de eritropoietina. ${ }^{16-18}$ Além disso, o relato de fraqueza foi o sintoma diretamente associado com a presença de anemia. Nos idosos a anemia é pouco tolerada, visto que a diminuição dos níveis de oxigênio nos tecidos leva a um aumento do débito cardíaco, tornando-os mais vulneráveis às manifestações clínicas de fraqueza. ${ }^{19}$

O uso de medicamentos na população idosa é frequente, e isso tem impacto nas condições clínicas, econômicas e de segurança. ${ }^{20} \mathrm{Em}$ 89,2\% dos idosos foi verificada a utilização de algum tipo de medicamento no seu dia a dia. Dentre as classes farmacológicas implicadas no desenvolvimento de anemia, os anti-inflamatórios não esteroidais (AINES) se destacam pela conhecida associação entre seu uso e o desenvolvimento de úlcera gástrica, que leva a perda sanguínea crônica e consequente anemia $;^{20} \mathrm{o}$ presente estudo, no entanto, não demonstrou tal associação.

A utilização de medicamentos antianêmicos é uma alternativa no tratamento da anemia, sendo identificado que os idosos com anemia utilizavam com maior frequência esse medicamento. Em contrapartida, do total de medicamentos, seu uso era pequeno e não abrangia toda a população anêmica, semelhante ao encontrado por Flores \& Benvegnú. ${ }^{20} \mathrm{O}$ não tratamento da anemia no idoso pode acarretar mais complicações clínicas, já que, pelo próprio processo de envelhecimento, esses indivíduos apresentam maior dificuldade de recuperação e maior vulnerabilidade a essa condição. ${ }^{8}$

Os tipos de anemia mais encontrados nos idosos são a anemia da doença crônica e por deficiência de ferro. ${ }^{21}$ No presente estudo, a grande maioria dos idosos apresentou anemia 
normocítica e normocrômica, o que é sugestivo de anemia por doença crônica. Em 50\% dos casos de anemia da doença crônica, é encontrada alteração hematimétrica hipocromia, ${ }^{22}$ resultado que correspondeu ao segundo achado hematimétrico mais frequente nos idosos anêmicos, sugerindo associação com a anemia por deficiência de ferro. Nas doenças crônicas, pode haver diminuição dos níveis plasmáticos de ferro, não por deficiência, mas por um bloqueio do fluxo do ferro dos macrófagos para o plasma. ${ }^{22}$ Esses dados são compatíveis com os descritos por Sgnaolin et al., ${ }^{8}$ segundo os quais $46,0 \%$ da população estudada apresentavam anemia normocítica e normocrômica, seguidos de 26,0\% de anemia normocítica e hipocrômica.

Por ser um estudo transversal, suas limitações estão relacionadas ao fato de os dados sobre o diagnóstico das anemias terem sido realizados baseando-se apenas no exame laboratorial e não por meio do diagnóstico clínico. Cabe ressaltar que todos esses resultados laboratoriais foram encaminhados para a ESF correspondente de cada idoso, onde eles tiveram acompanhamento médico, quando necessário.

\section{CONCLUSÃO}

Os dados sobre a prevalência da anemia podem variar bastante, dependendo da localização e da população estudada. O presente estudo demonstrou baixa prevalência de anemia nos idosos de Porto Alegre-RS. Inúmeros são os fatores desencadeantes dos baixos níveis de hemoglobina, tais como: faixa etária, cor, renda mensal familiar, escolaridade, cuidados e doenças associadas. $\mathrm{O}$ melhor diagnóstico perante os baixos níveis de hemoglobina deve ser realizado, e a caracterização da anemia deve ser cuidadosamente avaliada. A anemia não deve ser uma condição negligenciada durante o envelhecimento, bem como as inúmeras doenças que acometem essa população e, portanto, merece atenção clínica e tratamento adequado.

\section{REFERÊNCIAS}

1. World Health Organization. Iron deficiency anaemia: assessment, prevention and control: a guide for programme managers. WHO: Geneva; 2001.

2. Beghé C, Wilson A, Ershler WB. Prevalence and outcomes of anemia in geriatrics: a systematic review of the literature. Am J Med 2004;16(7 Suppl 1):3-10.

3. Guralnik JM, Ershler WB, Schrier SL, Picozzi VJ. Anemia in the elderly: a public health crisis in hematology. Hematology 2005;2005(1):528-32.

4. Artz AS, Fergusson D, Drinka PJ, Gerald M, Gravenstein S, Lechich A, et al. Prevalence of anemia in skilled-nursing home residents. Arch Gerontol Geriatr 2004;39(3):201-6.

5. Nóbrega OT, Karnikowski MGO. A terapia medicamentosa no idoso: cuidados na medicação. Rev Ciênc Súde Coletiva 2005;10(2):309-13.

6. Lippi G, Franchini M, Salvagno GL, Montagnana M, Targher G, Guidi GC. Determinants of anaemia in the very elderly: a major contribution from impaired renal function? Blood Transfus 2010;8(1):44-8.

7. Guralnik JM, Eisenstaedt RS, Ferrucci L, Klein HG, Woodman RC. Prevalence of anemia in persons 65 years and older in the United States: evidence for a high rate of unexplained anemia. Blood 2004;104(8):2263-8.

8. Sgnaolin V, Engroff P, Ely LS, Schneider RH, Schwanke CH, Gomes I, et al. Hematological parameters and prevalence of anemia among freeliving elderly in south Brazil. Rev Bras Hematol Hemoter 2013;35(2):115-8.

9. Travassos C, Viacava F. Acesso e uso de serviços de saúde em idosos residentes em áreas rurais, Brasil, 1998 e 2003. Cad Saúde Pública 2007;23(10):2490-502.

10. Bang SM, Lee JO, Kim YJ, Lee KW, Lim S, Kim $\mathrm{JH}$, et al. Anemia and activities of daily living in the Korean urban elderly population: results from the Korean Longitudinal Study on Health and Aging (KLoSHA). Ann Hematol 2013;92(1):59-65.

11. Culleton BF, Manns BJ, Zhang J, Tonelli M, Klarenbach S, Hemmelgarn BR. Impact of anemia on hospitalization and mortality in older adults. Blood 2006;107(10):3841-6. 
12. Haverkate M, Smits J, Meijerink H, Van der Ven A. Socioeconomic determinants of haemoglobin levels of african women are less important in areas with more health facilities: a multilevel analysis. J Epidemiol Community Health 2014;68(2):116-22.

13. Simonetti JP, Ferreira JC. Estratégias de coping desenvolvidas por cuidadores de idosos portadores de doença crônica. Rev Esc Enferm USP 2008;42(1):19-25.

14. Instituto Brasileiro de Geografia e Estatística. Síntese de indicadores sociais: uma análise das condições de vida da população brasileira 2013 [Internet]. Brasília, DF: IBGE; 2013 [acesso em 10 jun 2013]. (Estudo e Pesquisas Demográfica e Socioeconômica, no 32). Disponível em: ftp.ibge.gov.br/Indicadores_Sociais/ Sintese_de_Indicadores_Sociais_2013/SIS_2013.pdf

15. Noronha KVMS, Andrade MV. Desigualdades sociais em saúde e na utilização dos serviços de saúde entre os idosos na América Latina. Rev Panam Salud Publica 2005;17(5/6):410-8.

16. Das KC, Mukherjee M, Sarkar TK, Dash RJ, Rastogi GK. Erythropoiesis and erythropoietin in hypo- and hyperthyroidism. J Clin Endocrinol Metab 1975;40(2):211-20.

17. Horton L, Coburn RJ, England JM, Himsworth RL. The haematology of hypothyroidism. Q J Med 1975;45(1):101-24.

18. Utiger RD. Hypothyroidism. In: De Groot LJ, editor. Endocrinology. vol.1. Philadelphia: W.B. Saunders; 1995. p.752-68.

19. Hoffbrand V, Moss P, Pettit J. Essential Haematology. Oxford: Wiley-Blackwell; 2006.

20. Flores VB, Benvegnú LA. Perfil de utilização de medicamentos em idosos da zona urbana de Santa Rosa, Rio Grande do Sul, Brasil. Cad Saúde Pública 2008;24(6):1439-46.

21. Barbosa DL, Arruda IKG, Diniz AS. Prevalência e caracterização da anemia em idosos do Programa de Saúde da Família. Rev Bras Hematol Hemoter 2006;28(4):288-92.

22. Cançado RD, Chiattone CS. Anemia de Doença Crônica. Rev Bras Hematol Hemoter 2002;24(2):127-36. 